\title{
Current-voltage characteristics of polar heterostructure junctions
}

\author{
Madhusudan Singh ${ }^{\text {a) }}$ and Jasprit Singh \\ Department of Electrical Engineering and Computer Science, University of Michigan, \\ Ann Arbor, Michigan 48109 \\ Umesh Mishra \\ Department of Electrical and Computer Engineering, University of California, \\ Santa Barbara, California 93106
}

(Received 7 May 2001; accepted for publication 16 November 2001)

\begin{abstract}
We report calculations that show that a metal-polar semiconductor heterostructure can exhibit highly controllable nonlinear current-voltage characteristics. A change in barrier thickness can alter the characteristics from Schottky-like to ohmic in different bias regimes. The origin of these unusual effects is a large electric field $\left(>10^{6} \mathrm{~V} / \mathrm{cm}\right)$ and high sheet charge $\left(\sim 10^{13}-10^{14} \mathrm{~cm}^{-2}\right)$ without doping, in the polar heterostructure. Theoretical calculation of the tunneling current density in these systems is done in this work. The results indicate that very interesting nonlinear behavior is shown by these systems, even in the undoped case. The choice of suitable compositions of the materials and thicknesses can be used to tailor devices with desired characteristics. (C) 2002 American Institute of Physics. [DOI: 10.1063/1.1434542]
\end{abstract}

\section{INTRODUCTION}

Group III-Nitride semiconductors have attracted wide attention recently in view of their application in high power devices and optoelectronic devices with wavelengths ranging from the red into the ultraviolet. ${ }^{1-9}$ It is known that there is a large spontaneous polarization in the nitrides. Additionally, the piezoelectric effect is also very strong. As a result, in heterostructures such as $\mathrm{AlGaN} / \mathrm{GaN}$ there is a large fixed polar charge at heterointerfaces. ${ }^{10}$ This charge can be exploited to introduce very large electric fields (and band bending) in heterostructures. The fixed polar charge for all purposes acts as a doped charge and can be exploited to create mobile carrier density and other phenomena associated with dopants. Indeed, in AlGaN/GaN heterojunction field effect transistors, this feature is exploited to create very high sheet charge devices without doping the device.

In this article, we examine how the polar charge and the resultant band bending can be used to tailor tunneling probabilities and current-voltage (I-V) relations in a metalheterostructure junction. There has been very little examination of vertical transport in such structures and it is usually expected that due to the large band gap of the materials, metal-semiconductor junctions would show Schottky behavior with large turn-on voltages. This is a correct expectation except for the cases where the distance between the metal and the heterointerface becomes very small (several tens of Angstroms). In this article, we will examine this regime. We find that by carefully tailoring the composition and thickness, it is possible to tailor the $\mathrm{I}-\mathrm{V}$ characteristics. It is possible to have linear (ohmic) characteristics as well as highly nonlinear ones.

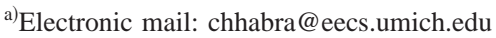

In the next section, we will discuss the formalism that forms the basis of this work. Results are presented in Sec. III. We conclude in Sec. IV.

\section{FORMALISM}

In this article, we will examine the band profiles and $\mathrm{I}-\mathrm{V}$ relations for a number of polar heterostructures. To address the problem, we need several ingredients: (i) A model for the material parameters describing not only the carrier masses, band line-ups etc. but also the polar charge at the interfaces. Such information is now available for the nitrides, and (ii) a self-consistent approach to the solution of the Schrödinger equation and Poisson equation. We have published details of such a model previously; (iii) a model for the tunneling current through the heterostructure.

\section{A. Material parameters}

For completeness we will summarize the value of the polar charges in the nitride system. There are two sources of polar charge at the heterointerfaces of nitride heterostructures. The strain due to coherent epitaxy (below critical thickness) results in a piezoelectric effect induced charge. In addition, the shift in the cation and anion sublattices in the grown crystal leads to spontaneous polarization. ${ }^{10,11}$

The piezoelectric polarization produced in growth of $\mathrm{Al}_{x} \mathrm{Ga}_{1-x} \mathrm{~N}$ on $\mathrm{GaN}$ is,

$$
P_{p z}(x)=\left(-3.2 x-1.9 x^{2}\right) \times 10^{-6} \mathrm{Ccm}^{-2} .
$$

In addition to the polarization induced by strain, the cation and anion sublattices are spontaneously displaced with respect to each other producing an additional polarization. For heterostructures, the difference of the spontaneous polarization appears at the interfaces. The values of the spontaneous polarization ${ }^{3}$ are:

$$
\text { InN: } \quad P_{\mathrm{sp}}=-3.2 \times 10^{-6} \mathrm{C} / \mathrm{cm}^{2}
$$


GaN: $\quad P_{\mathrm{sp}}=-2.9 \times 10^{-6} \mathrm{C} / \mathrm{cm}^{2}$

AlN: $\quad P_{\mathrm{sp}}=-8.1 \times 10^{-6} \mathrm{C} / \mathrm{cm}^{2}$.

The two effects described become important when heterostructures are grown. In the case of spontaneous polarization, the presence of a heterostructure between materials with different spontaneous polarization values causes a net charge at the interface which causes built-in electric fields in the structure. Similarly, in case the lattice constants of the components of the heterostructure are different, the resulting strain present (assuming minimal dislocation generation) causes charges at the interfaces due to the piezoelectric effect.

The magnitude and direction of the electric fields associated with spontaneous polarization and piezoelectric effect depend on the substrate, the growth orientation, and the nature of the surface (cation terminated or anion terminated). For the results given here, we will discuss the most common growth conditions employed for the nitride systems where growth is on sapphire and is along the (0001) direction with Ga terminated surface. The effective substrate is defined by the a thick buffer that is grown on the starting substrate (sapphire). Once dislocations are generated, the thick buffer forms its own lattice and acts as a substrate for the next layers as long as the growth is coherent i.e., buffer thickness is small enough to generate no dislocations. The buffer now grows with a lattice structure which fits the in-plane lattice of the substrate and has an out-of-plane lattice constant defined by total energy minimization. Thus, a compressive strain in the plane of growth causes a tensile strain out of plane.

Consider for example a case where the effective substrate is $\mathrm{GaN}$ and an $\mathrm{Al}_{x} \mathrm{Ga}_{1-x} \mathrm{~N}$ epitaxial layer is grown coherently. The polarization is found to have the value. ${ }^{12}$

$$
\begin{aligned}
P(x)= & P_{p z}+P_{s p} \\
= & \left(-3.2 \times x-1.9 x^{2}\right) \times 10^{-6} \mathrm{C} / \mathrm{cm}^{2} \\
& -5.2 \times 10^{-6} \times \mathrm{C} / \mathrm{cm}^{2} .
\end{aligned}
$$

We see that in this system the effects arising from piezoelectric effect and spontaneous polarization mismatch are comparable. Note that the two effects can have opposite directions as well depending on the surface termination conditions and the lattice mismatch between the epitaxial layer and the effective substrate. The electric field associated with the polarization given is

$$
F(x)=\left(-9.5 x-2.1 x^{2}\right) \mathrm{MV} / \mathrm{cm} .
$$

We see that the built-in field and sheet charge values are very large. It is easy to produce fields around $10^{6} \mathrm{~V} / \mathrm{cm}$ and charge density around $10^{13} \mathrm{~cm}^{-2}$.

\section{B. Charge control}

The detailed formalism has been described earlier. ${ }^{13,14}$ In the self-consistent approach, we solve the Schrödinger equation for the wave functions which yield the confined charge terms. The Poisson equation is then solved for potential that is fed back into the Schrödinger equation until the solution goes to convergence. This provides us the subband levels, their occupation, and wave functions.

We model the slope at the AlN/GaN (and AlGaN/GaN) interface of the conduction band to be discontinuous. In reality, there is a certain nonzero penetration depth of the charge into the substrate. This has two effects: (1) Reducing the amount of charge at the interface, and (2) Effectively increasing the barrier thickness from the metal contact to the region of quantum confinement.

The peak of the ground state wave function is located some distance away from the material interface of AlN/GaN. Since the amount of charge induced in the two-dimensional electron gas (2DEG) depends upon the thickness of the barrier layer, penetration depth also varies with thickness of barrier layer. From our calculations, the width of the potential well (2DEG, ground state) for the $50 \AA \mathrm{AlN}$ barrier is about $10 \AA$. Since the peak of the wave function is located within it, this is the upper limit on the penetration depth. Since the increase in barrier thickness takes place over the entire interface region between AIN and GaN, and the band gap difference between $\mathrm{AlN}$ and GaN is large, this is not expected to distort the potential profile from the discontinuous case appreciably. Furthermore, the penetration of the wave function into the AlN barrier layer is minimal owing to the large band gap difference between AlN and GaN.

\section{Tunneling calculation}

Once the band profile is known we use the WentzelKramers-Brillouin (WKB) method to obtain the tunneling probability $^{14}$ from the metal to the GaN channel. To obtain the tunneling current, we consider the density of states in the GaN substrate. The density of states can be divided into two regimes:

(1) Within the region of quantum confinement, which is the two dimensional density of states. The corresponding current density $J_{2 \mathrm{D}}$ is defined in Eq. (5), and

(2) At energies greater than confinement energy, we have a three-dimensional density of states. The corresponding current density $J_{3 \mathrm{D}}$ is defined in Eq. (6). Thermionic emission is accounted for by extending the range of energies above the heights of relevant barriers.

The metal contact is considered to be an infinite source of electrons. The expression for the tunneling current is

$$
\begin{aligned}
J_{\text {tunnel }} & =J_{2 \mathrm{D}}+J_{3 \mathrm{D}}, \\
J_{2 \mathrm{D}}= & q \sum_{n=1}^{n=n_{2 \mathrm{D}}} \frac{v\left(E_{n}, E_{f}\right) D_{2 \mathrm{D}}\left(E_{n}\right)}{L_{n}} \\
& \times \int_{0}^{\infty}\left(f\left(E_{n}+E_{t}, E_{f s}, T\right)-f\left(E_{n}+E_{t}, E_{f m}, T\right)\right. \\
& \times T\left(E_{n}, E_{t}\right) d E_{t},
\end{aligned}
$$



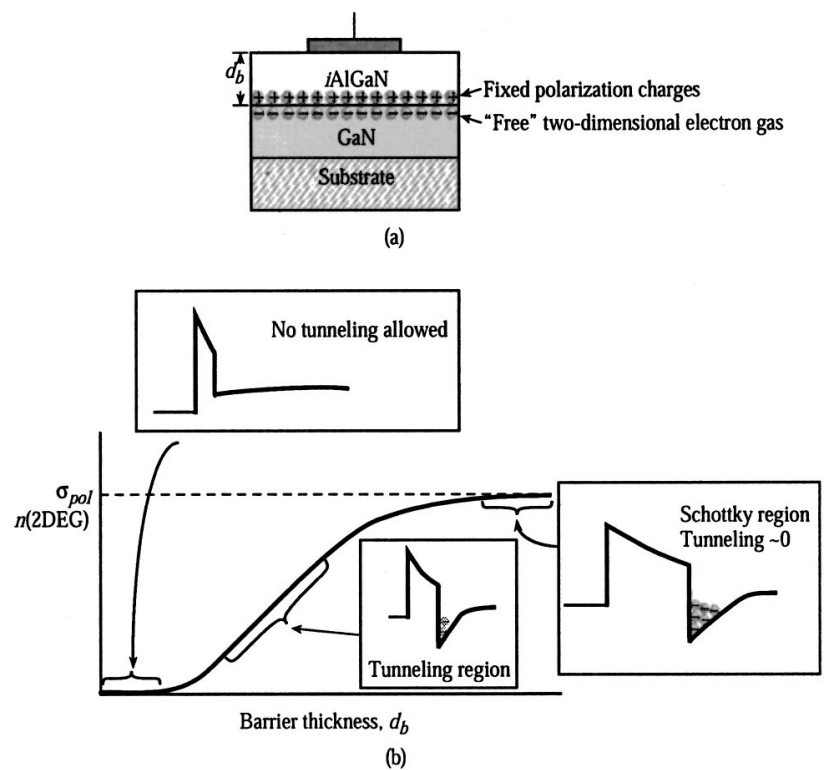

FIG. 1. A schematic of the metal-AlGaN/GaN is shown. In (a), we show the fixed polarization charge and the induced 2DEG. In the lower part, we show how a variation of the AlGaN thickness influences the 2DEG and tunneling. The back contact is made to the 2DEG directly.

$$
\begin{aligned}
J_{3 \mathrm{D}}= & q \int_{E_{n_{2 \mathrm{D}}}}^{\infty} \int_{0}^{\infty} v\left(E_{l}, E_{f}\right) D_{1 \mathrm{D}}\left(E_{l}\right) D_{2 \mathrm{D}}\left(E_{t}\right) \\
& \times\left(f\left(E_{l}+E_{t}, E_{f s}, T\right)-f\left(E_{l}+E_{t}, E_{f m}, T\right)\right. \\
& \times T\left(E_{l}, E_{t}\right) d E_{t} d E_{l},
\end{aligned}
$$

where $n_{2 \mathrm{D}}$ is the total number of bound levels in the 2DEG. $E_{f}$ is given by

$$
E_{f}=\min \left(E_{f s}, E_{f m}\right) \text {. }
$$

The velocity of carriers in these equations is a function of $E_{n}$ and $E_{l}$ in addition to $E_{f}$ as defined. Thus, the velocity is a function of the final energies over which the expressions are being summed or integrated. $E_{f}$ is defined this way to ensure correct integration ranges for the carrier current.

Calculation of the tunneling current is carried out using MATHEMATICA. Monte Carlo integration is chosen for its faster evaluation time.

\section{RESULTS}

To illustrate how mobile sheet charge density at an interface and tunneling probability varies with barrier film thickness, we show a schematic of a typical band profile for a heterostructure with polar charge in Fig. 1. For very small thicknesses, the probability of tunneling through the barrier is high, but the charge control model shows that there is very little carrier population in the 2DEG. In this case, there are no allowed states to tunnel into from the metal and as a result there is no tunneling. For large thicknesses, there is an increase in the number of available states, but the WKB tunneling probability is suppressed. Thus, we have a trade off that optimizes the tunneling current. In the intermediate region, the barrier thickness is large enough to allow 2DEG and small enough to allow tunneling through the barrier.

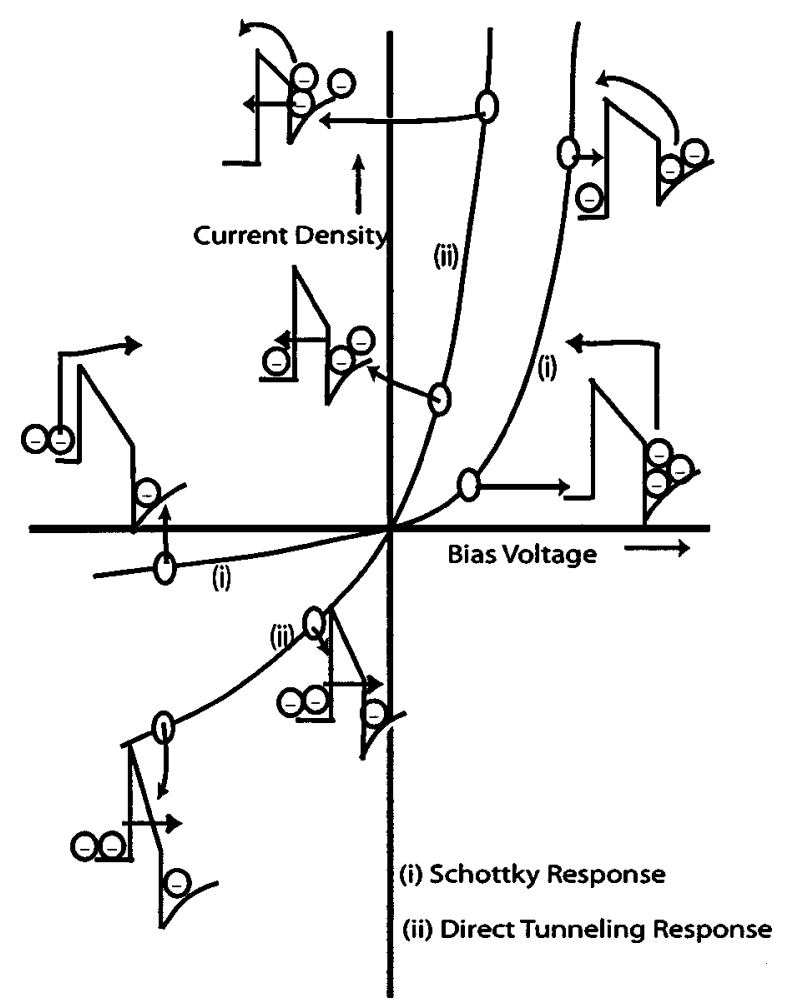

FIG. 2. Variation of nature of the I-V characteristic with thickness of epitaxial layer. For representative bias points on either characteristic, band profiles of the conduction band are shown. The arrows across the barriers indicate directions of electron flow. Thermionic emission and direct tunneling are both exhibited. In the reverse bias region for either characteristic, the barrier for thermionic emission remains roughly constant.

Moreover, the precise shape of the $\mathrm{I}-\mathrm{V}$ characteristic is found to be a strong function of barrier thickness. We have summarized the two kinds of behavior obtained in the two thickness regimes, in Fig. 2. Direct tunneling becomes feasible for smaller thicknesses and maintains a relatively high current density even in the reverse bias region. The back contact to the device is made to the 2DEG directly. The effect of thermionic emission is shown in the characteristic Fig. 2 (i).

The barrier to current flow in the reverse bias is roughly constant, while it changes quickly with forward bias. This means that the current increase per unit bias change (or differential conductivity) in the reverse bias region is smaller than the differential conductivity for forward bias region. This feature is borne out by all of our results.

We examine two categories of heterostructures in these simulations. (i) In the first category, the alloy AlGaN is the barrier and $\mathrm{GaN}$ is the substrate and active region, and (ii) in the second case, pure AlN forms the barrier. The exact compositions used are (1) $\mathrm{Al}_{0.28} \mathrm{Ga}_{0.72} \mathrm{~N} / \mathrm{GaN}$. The GaN substrate is taken to be undoped. (2) AlN/p-GaN. The doping in substrate $\mathrm{GaN}$ is taken to be $1 \times 10^{17} \mathrm{~cm}^{-3}$. The inclusion of a small $p$-doping in GaN has essentially no effect on the results and is included only since as grown $\mathrm{GaN}$ samples often have a small $p$-type doping level.

Except in the second case, there is no doping in the structure and the 2DEG is induced solely as a result of the fixed polarization charge. 


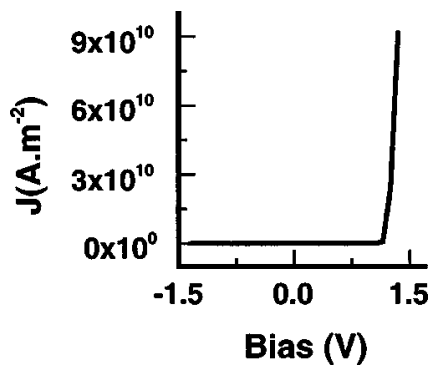

FIG. 3. Schottky behavior of $\mathrm{Al}_{0.28} \mathrm{Ga}_{0.72} \mathrm{~N} / \mathrm{GaN}$ junction with a large barrier thickness.

As the layer thicknesses are altered, the fixed polar charge at the relevant interface remains constant. However, the free carrier density it induces at the interface changes due to changes in the surface potential. This implies that the bending of bands either reduces or enhances, depending upon the applied bias. This has direct consequences for the number of available two-dimensional (2D) states in the 2DEG. This, as we shall see now, determines the general shape of the $\mathrm{I}-\mathrm{V}$ characteristic.

As noted earlier, at too small a barrier thickness the 2D channel gets depleted and the energy levels in the heterostructure are above the energy levels near the metal Fermi level.

We start with results for the $\mathrm{Al}_{0.28} \mathrm{Ga}_{0.72} \mathrm{~N} / \mathrm{GaN}$ system. Due to the polar fixed charge at large AlGaN thicknesses, $2 \mathrm{DEG}$ is induced at the interface with a density of 3 $\times 10^{13} \mathrm{~cm}^{-2}$. Figure 3 demonstrates the essential Schottky nature of the barrier at very large thicknesses. As the applied bias approaches the Schottky barrier height assumed at the metal-AlGaN interface $(1.4 \mathrm{eV})$, the current experiences an
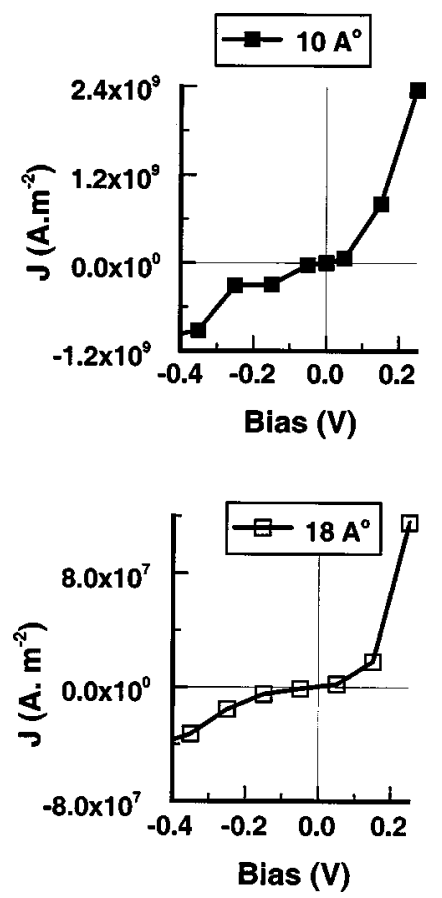

FIG. 4. Set of results for the device structure $\mathrm{Al}_{0.28} \mathrm{Ga}_{0.72} \mathrm{~N} / \mathrm{GaN}$. There is an initial slight decrease in the tunneling current with increase in thickness (from 10 to $18 \AA$.)
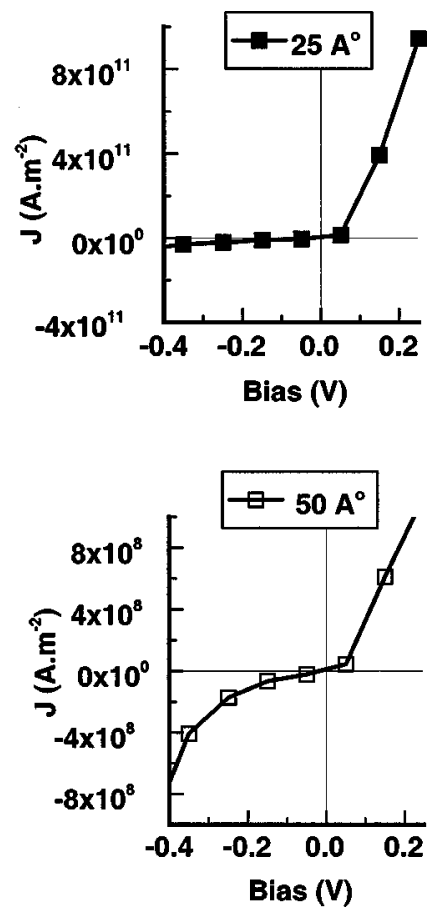

FIG. 5. Second part of results for the device structure $\mathrm{Al}_{0.28} \mathrm{Ga}_{0.72} \mathrm{~N} / \mathrm{GaN}$. The peak of the current capability is achieved for $25 \AA$. There is a decrease in the tunneling due to large barrier thickness ( $50 \AA$ ).

extremely rapid increase. The device turn on occurs at current densities of about $10^{8}-10^{9} \mathrm{~A} \mathrm{~m}^{-2}$. This happens at about $1.1 \mathrm{~V}$ in Fig. 3.

In Figs. 4 and 5, we show results for the I-V characteristics as the AlGaN thickness is reduced. We can see that when the barrier thickness decreases to $\sim 25 \AA$, the structure starts to turn on (as defined earlier) at very small biases. Notice also that the current values are small at thicknesses larger than $25 \AA$ and smaller than $25 \AA$. A few features of this study are worth noting.

(1) The characteristic is nonlinear around zero bias. This may be understood in terms of the asymmetry in the density of states on either side of the barrier.

(2) The characteristic tends to linearize in different bias ranges somewhat as barrier thickness is raised. This is a reflection of the increase in carrier density in the 2DEG of the well. This makes the carrier distributions on either side of the potential well less asymmetric. At the same time, the reduction in the tunneling probability causes a damping in the relative differences in the characteristic.

(3) If the barrier thickness is made too large, (see Fig. 5-50 $\AA$ ), the magnitude of the tunneling current reduces.

(4) The turn on is faster for lower thicknesses. This can have implications for the transient response of the device.

We next examine the AlN/GaN structure. The total polar charge is about $6 \times 10^{13} \mathrm{~cm}^{-2}$ in this case.

Figure 6 again demonstrates the Schottky nature of the barrier. The current is suppressed somewhat owing to the increased height of the barrier. This means that the turn-on voltage in this case is higher than in the earlier case. The device now turns on at about $2.0 \mathrm{~V}$. An interesting compari- 

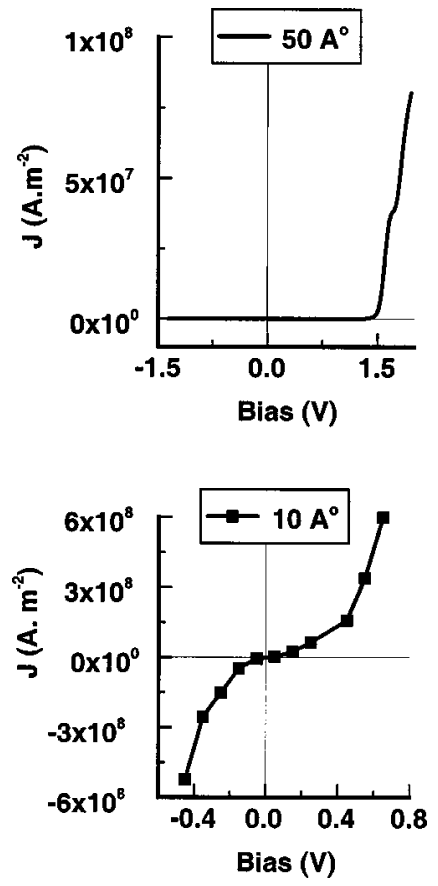

FIG. 6. Set of results for the device structure AlN/p-GaN. GaN substrate is doped $1 \times 10^{17} \mathrm{~cm}^{-3}$. Schottky barrier response is evident for the larger thickness (50 $)$ ), while direct tunneling comes into play at lower thickness for $10 \AA$.

son may be made with second part of Fig. 6 wherein we have presented the I-V characteristic for AlN thickness of $10 \AA$. The shape of the characteristic is different as well as the absolute magnitude of the current density is higher for this case. However, experimentally, it is difficult to grow AlN thicknesses of $10 \AA$ in a controlled fashion. The change over from Schottky to the direct tunneling regime, as discussed earlier, is apparent.

If we compare Figs. 6 with 4 and 5, the overall behavior looks less linear for reverse bias. This is due to the increased asymmetry in the charge distribution due to higher polarization charge at the interface.

\section{CONCLUSIONS}

In this article, we have examined vertical transport in metal-polar heterostructure junctions. In these structures, the fixed polar charge causes band bending and free charge accumulation effects that normally require doping. We have discussed our formalism which allows us to self-consistently calculate the band profile in polar heterostructures and then calculate $\mathrm{I}-\mathrm{V}$ relations.

We find that by carefully designing the thickness of the epitaxial layer, several interesting responses can be obtained. We have examined these regimes for two categories of heterostructures. When the epitaxial layer thickness is large, we see Schottky barrier response with a cut in voltage at a forward bias close to the Schottky barrier height. As the barrier thickness is decreased, the $\mathrm{I}-\mathrm{V}$ relation can approach ohmic behavior in certain bias regimes and also show a diode-like behavior with a very small turn-on voltage. Turn-on voltage can be an order of magnitude smaller that the Schottky barrier height. When the barrier thickness is very small, the current flow is once again described by Schottky-like behavior.

If we consider issues related to device design, we identify that within limits, increasing the barrier thickness linearizes the characteristic in different voltage ranges. This also has the effect of reducing the magnitude of the reverse current as tunneling probability is suppressed. However, increasing barrier thickness indefinitely leads to a very low current. As shown in Fig. 5, certain intermediate thicknesses can also yield a characteristic which is reasonably linear in certain bias ranges. In a nutshell, the current carrying capability of the device as well as its shape is optimized with respect to two requirements-high tunnel probability (decreased with increasing barrier thickness) and high density of states in 2DEG (increased with increasing barrier thickness).

The theoretical studies presented here have not been verified experimentally as yet. However, given their potential applications, we expect experimental groups will examine the polar heterostructure junction.

\section{ACKNOWLEDGMENTS}

The work at the University of Michigan has been supported by Grant Nos. F001681 (the POLARIS program) and F000629 from the U.S. Office of Naval Research. The work at UC Santa Barbara has been supported by the ONR POLARIS program.

${ }^{1}$ I. Akasaki, H. Amano, S. Nagahama, T. Tanaka, and M. Koike, Jpn. J. Appl. Phys., Part 2 34, 1517 (1995).

${ }^{2}$ S. Nakamura, M. Senoh, S. Nagahama, N. Iwasa, T. Yamada, T. Matsushita, Y. Sugimoto, and H. Kiyoku, Appl. Phys. Lett. 69, 3034 (1996).

${ }^{3}$ G. E. Bulman, K. Doverspike, S. T. Sheppard, T. W. Weeks, H. S. Kong, H. M. Dieringer, J. A. Edmond, J. D. Brown, J. T. Swindell, and J. F. Schetzena, Electron. Lett. 33, 1556 (1997).

${ }^{4}$ M. P. Mack, A. Abare, M. Aizcorbe, P. Kozodoy, S. Keller, U. K. Mishra, L. Coldren, and S. DenBaars, MRS Internet J. Nitride Semicond. Res. 2, 56 (1997).

${ }^{5}$ O. Aktas, Z. F. Fan, A. Botchkarev, S. N. Mohammad, M. Roth, T. Jenkins, L. Kehias, and H. Morkoc, IEEE Electron Device Lett. 18, 293 (1997).

${ }^{6}$ M. S. Shur and M. A. Khan, MRS Bull. 22, 44 (1997).

${ }^{7}$ Y. F. Wu, S. Keller, P. Kozodoy, B. P. Keller, P. Parikh, D. Kapolnek, S. P. DenBaars, and U. K. Mishra, IEEE Electron Device Lett. 18, 290 (1997).

${ }^{8}$ U. K. Mishra, Y. F. Wu, B. P. Keller, S. Keller, and S. P. DenBaars, IEEE Trans. Microwave Theory Tech. 46, 756 (1998).

${ }^{9}$ R. Dimitrov, L. Wittmer, H. Felsl, A. Mitchell, O. Ambacher, and M. Stutzmann, Phys. Status Solidi A 168, R7 (1998).

${ }^{10}$ O. Ambacher, J. Smart, J. R. Shealy, N. G. Weimann, K. Chu, M. Murphy, W. J. Schaff, L. F. Eastman, R. Dimitrov, L. Wittmer, M. Stutzmann, W. Rieger, and J. Hilsenbeck, J. Appl. Phys. 85, 3222 (1999).

${ }^{11}$ F. Bernardini, V. Fiorentini, and D. Vanderbilt, Phys. Rev. B 56, 10024 (1997).

${ }^{12}$ I. P. Smorchkova, C. R. Elsass, J. P. Ibbetson, R. Ventury, B. Heying, P. Fini, E. Haus, S. P. DenBaars, J. S. Speck, and U. K. Mishra, J. Appl. Phys. 86, 4520 (1999).

${ }^{13}$ Y. Zhang and J. Singh, J. Appl. Phys. 85, 587 (1999).

${ }^{14}$ M. Singh, Y. Zhang, J. Singh, and U. Mishra, Appl. Phys. Lett. 77, 1867 (2000) 